\title{
Incubation of 2-methylisoborneol synthase with the intermediate analog 2-methylneryl diphosphate
}

\author{
Wayne KW Chou, Colin A Gould and David E Cane
}

Incubation of synthetic 2-methylneryl diphosphate (2-MeNPP, 10) with 2-methylisoborneol synthase (MIBS) gave a mixture of products that differed significantly from that derived from the natural substrate (E)-2-methylgeranyl diphosphate (3, 2-MeGPP). The proportion of (-)-2-methylisoborneol (1) decreased from 89 to $17 \%$ while that of 2-methylenebornane (4) increased from 10 to $26 \%$, with the relative yields of the isomeric homo-monoterpenes 2-methyl-2-bornene (5) and 1-methylcamphene (6) remaining essentially unchanged ( $<1 \%$ each), as determined by chiral GC-MS analysis. The majority of the product mixture resulting from the MIBS-catalyzed cyclization of 2-MeNPP (10) consisted of the anomalous monocyclic homo-monoterpenes $( \pm)$-2-methylllimonene $(15,39 \%)$ and 2-methyl- $\alpha$-terpineol $(13,10 \%)$, as well as the acylic derivatives 2 -methylnerol (11, 7\%) and 2-methyllinalool $(14,<1 \%)$. The steady-state kinetic parameters of the MIBS-catalyzed reaction, determined using $\left[1-{ }^{3} \mathrm{H}\right]-2-m e t h y l n e r y l ~ d i p h o s p h a t e(2-M e N P P)$, were $k_{\text {cat }} 0.0046 \pm 0.0003 \mathrm{~s}^{-1}, K_{\mathrm{m}} 18 \pm 6 \mu \mathrm{M}$ and $k_{\text {cat }} / K_{\mathrm{m}} 2.55 \times 10^{2} \mathrm{M}-1 \mathrm{~s}-1$. In comparison, the natural substrate $2-M e G P P$ had a $k_{\text {cat }} 0.105 \pm 0.007 \mathrm{~s}^{-1}, K_{\mathrm{m}} 95 \pm 49 \mu \mathrm{m}$ and $k_{\mathrm{cat}} / K_{\mathrm{m}} 1.11 \times 10^{3} \mathrm{M}-1 \mathrm{~s}-1$. Taken together with earlier X-ray crystallographic studies of MIBS, as well as previous investigations of the mechanistically related plant monoterpene cyclase, bornyl diphosphate synthase, these results provide important insights into the binding and cyclization of both native substrates and intermediates and their analogs.

The Journal of Antibiotics (2017) 70, 625-631; doi:10.1038/ja.2017.24; published online 1 March 2017

\section{INTRODUCTION}

Since the discovery of 2-methylisoborneol $(2-\mathrm{MIB}, 1)$ by Gerber in 1969 , there has been considerable interest in the detection, bioremediation and biosynthesis of this volatile, odiferous homo-monoterpene (Figure 1). ${ }^{1-4}$ Possessing a musty odor and muddy off-taste, as well as an extremely low threshold of detection by humans $\left(<10 \mathrm{ngl}^{-1}\right)$, 2-MIB is produced by a wide range of Gram-positive actinomycetes, myxobacteria and cyanobacteria, being second in occurrence only to the common odiferous sesquiterpene geosmin. ${ }^{5}$

The elucidation of the biosynthesis of 2-MIB at the molecular genetic and biochemical level was independently reported in 2008 by two research groups (Figure 1). ${ }^{6-8}$ The 2-MIB biosynthetic gene cluster in Streptomyces coelicolor was shown to harbor a terpene synthase gene (sco7700) translationally coupled to the gene for an S-adenosyl-L-methionine-dependent C-methyl transferase, (sco7701), as well as a third gene (sco7699) encoding a protein of still unknown function, annotated only as a nucleotide-binding protein. ${ }^{7}$ This three-gene biosynthetic operon is highly conserved across the genome sequences of more than three dozen bacterial species. ${ }^{3,5,6}$ The $S$-adenosyl-L-methionine-dependent geranyl diphosphate C-methyl transferase (GPPMT; Sco7701) was shown to catalyze the first step in the biosynthetic pathway in S. coelicolor, the electrophilic methylation of the olefinic C-2 position of geranyl diphosphate
(GPP, 2) to form (E)-2-methylgeranyl diphosphate (2-MeGPP, 3). ${ }^{7}$ Sco7700, 2-MIB synthase (MIBS), catalyzes the $\mathrm{Mg}^{2+}$-dependent multistep cyclization of the acyclic 2-MeGPP precursor to the bicyclic alcohol 2-MIB (1) which is accompanied by small amounts of the bicyclic homo-monoterpenes 2-methylenebornane (4), 2-methylbornene (5) and 1-methylcamphene (6). The only other enzyme known to utilize 2-MeGPP as substrate is the closely related 2-methylenebornane synthase (Pfl_1841) of Pseudomonas fluorescens PfO- $1 .{ }^{9}$ These findings were fully consistent with the results of independent whole-cell precursor incorporation experiments involving the feeding of $\left[\right.$ methyl- $\left.{ }^{13} \mathrm{C}\right]$ methionine and samples of deuterated mevalonate to the myxobacterium Nannocystis exedens and MS analysis of the resulting 2-MIB in the head-space volatiles. ${ }^{4}$

The proposed 2-MIB synthase mechanism is based on close analogy to the well-documented cyclization mechanisms established for a wide range of monoterpene synthases (Figure 2). ${ }^{10}$ MIBS initiates the electrophilic cyclization by ionizing the $\mathrm{Mg}^{2+}$-complexed substrate 2-MeGPP (3) to generate the corresponding allylic cation-pyrophosphate ion pair which then collapses to the transoid conformer of the allylic isomer (3R)-2-methyllinalyl diphosphate (2-MeLPP, 7). Rotation about the C-2,3-bond generates the cisoid conformer of 7, folded in the anti-endo-boat conformation, which then undergoes ionization and cyclization to generate the (4R)-2-methyl- $\alpha$-terpinyl 


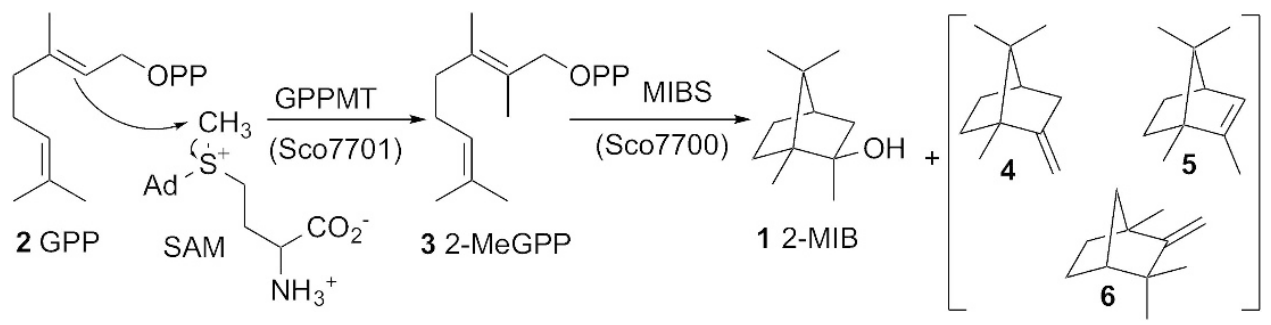

Figure 1 Biosynthesis of 2-methylisoborneol (1).

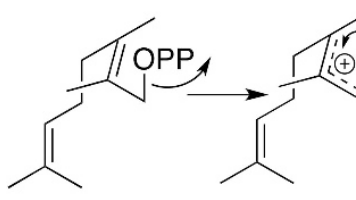

3 2-MeGPP

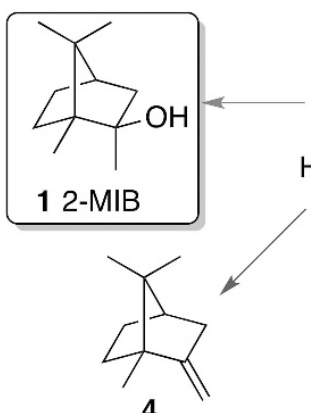

4
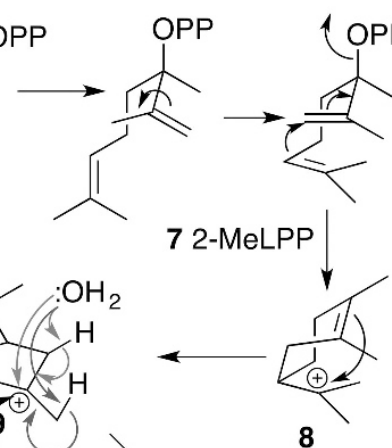

8

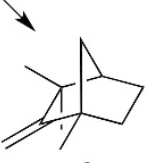

5
Figure 2 Mechanism of the 2-methylisoborneol synthase (MIBS)-catalyzed cyclization of 2-methylgeranyl diphosphate (3) to 2-methylisoborneol (1). A full color version of this figure is available at the The Journal of Antibiotics journal online.

cation (8). Electrophilic attack on the cyclohexenyl double bond of 8 generates the 2-methyl-2-bornyl cation (9), which is then quenched on the exo face by bound water to generate 2-MIB (1). Competing deprotonation of intermediate 9 or its proximal rearrangement product readily accounts for formation of the co-products 4, 5 and 6. The MIBS cyclization mechanism, including the deduced stereochemistry and conformations of the various intermediates, closely resembles that firmly established for (+)-bornyl diphosphate synthase/ $\alpha$-pinene synthase from Salvia officinalis which converts GPP (2) to (+)-bornyl diphosphate $(75 \%)$ as well as a mixture of the monoterpene hydrocarbons (+)- $\alpha$-pinene, $(+)$-camphene, $( \pm)$-limonene and terpinolene (25\% total olefins; Figure 3$).{ }^{11-13}$ There are of course two key differences between the reactions catalyzed by MIBS and by (+)-bornyl diphosphate synthase/ $\alpha$-pinene synthase: (1) MIBS cyclizes 2-MeGPP instead of GPP. Although MIBS can cyclize GPP to a mixture of monocyclic and bicyclic monoterpenes, the observed $k_{\text {cat }}$ is four orders of magnitude lower than that for the natural methylated substrate $2-\mathrm{MeGPP}^{7}$ (2) The final step in the MIBS-catalyzed cyclization cascade is the quenching of the 2-methyl-2-bornyl cation exclusively on the exo face by a bound water (Figure 2), while in the (+)-bornyl diphosphate synthase/ $\alpha$-pinene synthase-catalyzed reaction, the paired pyrophosphate ion is recombined with the bornyl cation exclusively by endo attack (Figure 3 ).

High-resolution crystal structures have recently been determined for MIBS in both an unliganded state and in complex with a number of
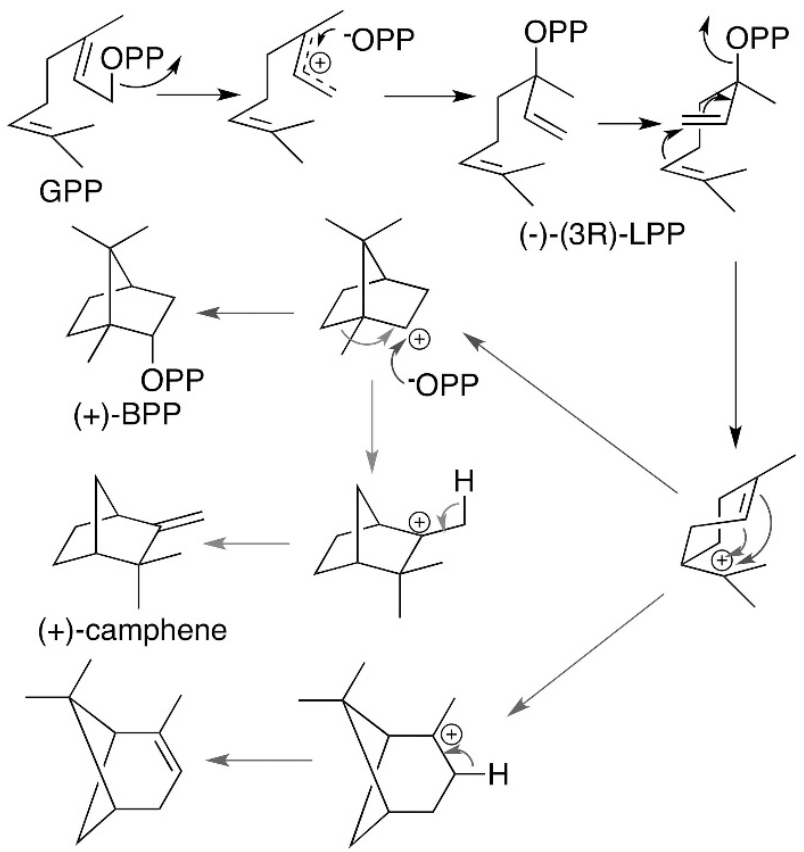

(+)- $\alpha$-pinene

Figure 3 Mechanism of bornyl diphosphate/ $\alpha$-pinene synthase. A full color version of this figure is available at the The Journal of Antibiotics journal online.

substrate or intermediate analogs. ${ }^{14,15}$ The $1.80-1.95 \AA$ structures of MIBS bound to geranyl-S-thiolodiphosphate and 2-fluorogeranyl diphosphate, respectively, show each analog coordinated with two $2 \mathrm{Mg}^{2+}$ ions and bound in extended conformations in which the 6,7-double bond of each analog is twisted away from the parallel relationship expected for the postulated anti-endo-boat conformation of the actual cyclizing cisoid (3R)-2-MeLPP intermediate (Figure 2). ${ }^{14}$ Although this deviation may simply be the result of crystallographic trapping of a thermodynamically stable, rather than a kinetically or mechanistically relevant conformer, more interestingly it reflects the fact that the required $180^{\circ}$ rotation of the 2-propenyl substituent about the C-2,3 bond of the anti-endo-boat conformer of the (3R)-2-methyllinalyl diphosphate intermediate is sterically forbidden by a clash with the 6,7-double bond that is augmented by the presence of the C-2-methyl substituent. It is thus probable that adoption of the endo-boat conformation takes place subsequent to conversion of the transoid to the cisoid conformer of 2-MeLPP. Such extended 6,7-double bond conformations of bound substrate analogs have previously been observed in the structures of a number of canonical monoterpene synthases. ${ }^{16,17}$ Also thought-provoking were the unexpected results from co-crystallization of MIBS with racemic 


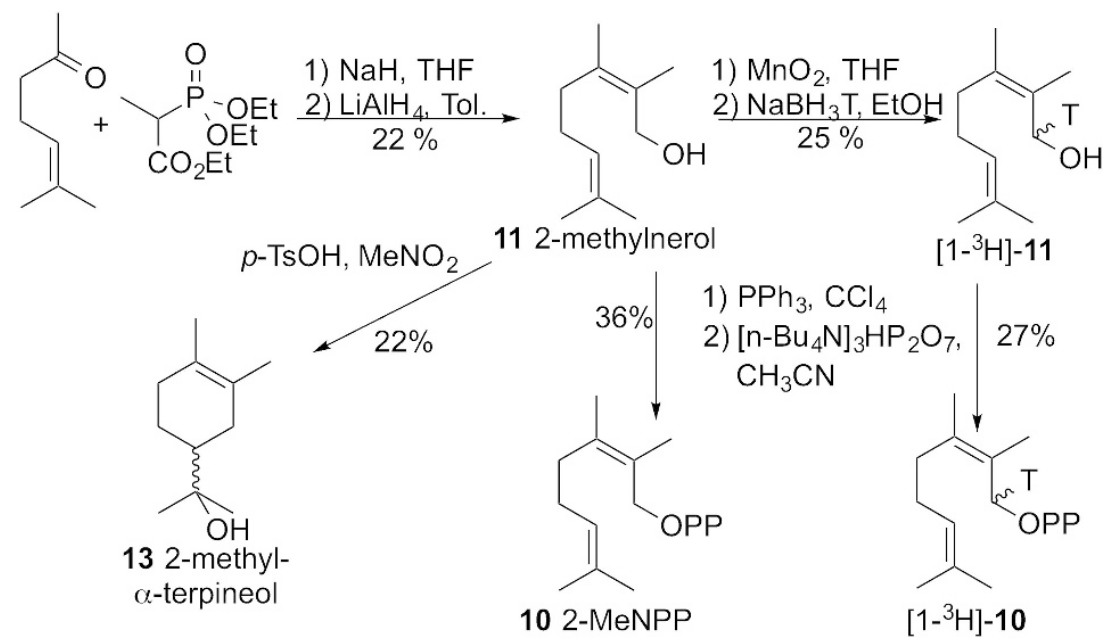

Figure 4 Synthesis of 2-methylneryl diphosphate (10, 2-MeNPP) and 2-methyl- $\alpha$-terpineol (13).

2-fluorolinalyl diphosphate (2-FLPP), which had been predicted to be an unreactive analog of the natural (3R)-2-methyllinalyl diphosphate intermediate due to the strongly electron-withdrawing 2 -fluoro substituent. The $2.00 \AA$ structure of the resulting complex revealed that the MIBS-bound 2-FLPP had undergone a reverse allylic rearrangement to generate bound 2-fluoroneryl diphosphate in complex with $2 \mathrm{Mg}^{2+}$ ions. ${ }^{15}$ Also unexpectedly, it was found that in solution MIBS converted the supposedly unreactive 2-FLPP to camphor, no doubt formed by cyclization of 2-FLPP and spontaneous elimination of hydrogen fluoride from enzymatically generated 2-fluoro-2-isoborneol. The observation of enzymegenerated 2-fluoroneryl diphosphate trapped within the active site of MIBS raises the question whether the cis geometric isomer of 2-FGPP is a true intermediate of the MIBS-catalyzed ionization and cyclization of 2-FLPP, the nominally unreactive analog of the natural intermediate 2-MeLPP (7), or merely an anomalous shunt metabolite of the reverse allylic diphosphate rearrangement. We have therefore now examined the incubation of MIBS with 2-MeNPP (10), the cis isomer of the natural substrate, 2-MeGPP (3), and report the results below.

\section{RESULTS}

Preparation of 2-methylneryl diphosphate (10) and ( \pm )-2-methyl$\boldsymbol{\alpha}$-terpineol (13)

To prepare 2-MeNPP (11), we used a modification of the previously reported synthesis of the trans isomer 2-MeGPP (3; Figure 4). ${ }^{7}$ Wadsworth-Horner-Emmons reaction of 6-methyl-5-hepten-2-one with triethyl 2-phosphonopropionate followed by $\mathrm{LiAlH}_{4}$ reduction of the resulting mixture of $Z$ and $E$ conjugated esters gave a 1:1 mixture of $(Z)$-2-methylnerol (11) and (E)-2-methylgeraniol (12) which were cleanly resolved by flash column chromatography (see Supplementary Information for details). The purified 2-methylnerol (11) was reacted with triphenylphosphine and $\mathrm{CCl}_{4}$ in an Appel reaction and the resulting 2-methylneryl chloride was then directly reacted without further purification with tris(tetrabutylammonium) hydrogen pyrophosphate to give 2-MeNPP (10). The corresponding $\left[1-{ }^{3} \mathrm{H}\right]-2-$ MeNPP $\left(\left[1-{ }^{3} \mathrm{H}\right]-10\right)$ was similarly prepared by oxidation of the mixture of 2-methylnerol and 2-methylgeraniol to the derived mixture of aldehydes, followed by reduction with $\mathrm{NaBH}_{3} \mathrm{~T}$ and chromatographic separation of the resulting mixture of $\left[1-{ }^{3} \mathrm{H}\right]-2$-methylnerol and $\left[1-{ }^{3} \mathrm{H}\right]-2$-methylgeraniol using $\mathrm{AgNO}_{3}$-impregnated silica gel.
For the planned enzyme incubations, we also needed an authentic reference sample of the homo-monoterpene alcohol $( \pm$ )-2-methyl- $\alpha$ terpineol (13), ${ }^{18}$ which was readily prepared by biomimeticallymodeled, $p$-TsOH-catalyzed solvolysis of 2-methylnerol (11) in nitromethane (Figure 4).

In vitro incubation of 2-methylneryl diphosphate with 2-methylisoborneol synthase

Before examining the cyclization of 2-MeNPP by methylisoborneol synthase, we carried out individual control incubations in the absence of enzyme with 2-MeGPP and 2-MeNPP and analyzed the pentane-soluble extracts by chiral GC-MS in order to identify and quantify the formation of $\mathrm{Mg}^{2+}$-catalyzed solvolysis products (Figures $5 \mathrm{a}$ and $\mathrm{b}$ ). The control incubation with 2-MeGPP produced only racemic $( \pm)$-2-methyllinalool (14). The exclusive formation of this tertiary allylic alcohol contrasts with the well-known $\mathrm{Mg}^{2+}$-catalyzed solvolysis of GPP itself, which typically gives a 3:1 mixture of linalool and geraniol. ${ }^{18}$ The preferred formation of 2-methyllinalool (14) by solvolysis of 2-MeGPP presumably reflects the higher free energy of 2-methylgeraniol due to the increased steric hindrance about the tetra-substituted double bond compared to geraniol. In contrast, the control $\mathrm{Mg}^{2+}$-catalyzed solvolysis of 2-MeNPP gave a mixture of three racemic products, consisting of acyclic $( \pm)$-2-methyllinalool (14) as well as the monocyclic ( \pm )-2-methyllimonene (15), and $( \pm)-2$-methyl- $\alpha$-terpineol (13; Figure $5 \mathrm{~b})$. The $\mathrm{Mg}^{2+}$-catalyzed formation of both $( \pm)-\mathbf{1 3}$ and $( \pm)-\mathbf{1 5}$ from 2-MeNPP, but not from 2-MeGPP, is consistent with the fact that the $(E)$-allylic diphosphate 2-MeGPP (3) is geometrically incapable of direct cyclization. As a positive control, incubation of 2-MeGPP (3) with 2-MIB synthase and chiral GC-MS analysis confirmed the formation of ( -$)$-2-MIB (1) as the major product (89\%) accompanied by minor amounts of enantiomerically pure 2-methylenebornane (4; 10\%), 1-methylcamphene $(6 ;<1 \%)$ and 2-methyl-2-bornene $(5 ;<1 \%)$, as previously observed (Figure $5 \mathrm{c}$ and Table 1$)^{6,7}$

Incubation of 2-MeNPP (10) with MIBS and analysis by chiral GC-MS revealed the production of the same group of homochiral bicyclic homo-monoterpenes, ( - )-2-MIB (1, 17\%), 2-methylenebornane (13, 26\%), 1-methylcamphene $(6)$ (<1\%) and 2-methyl-2bornene $(5)(<1 \%)$, but in both substantially different proportions and lower overall yield compared to 2-MeGPP (Figures 5d and 6). Significantly, four additional, previously unobserved products 

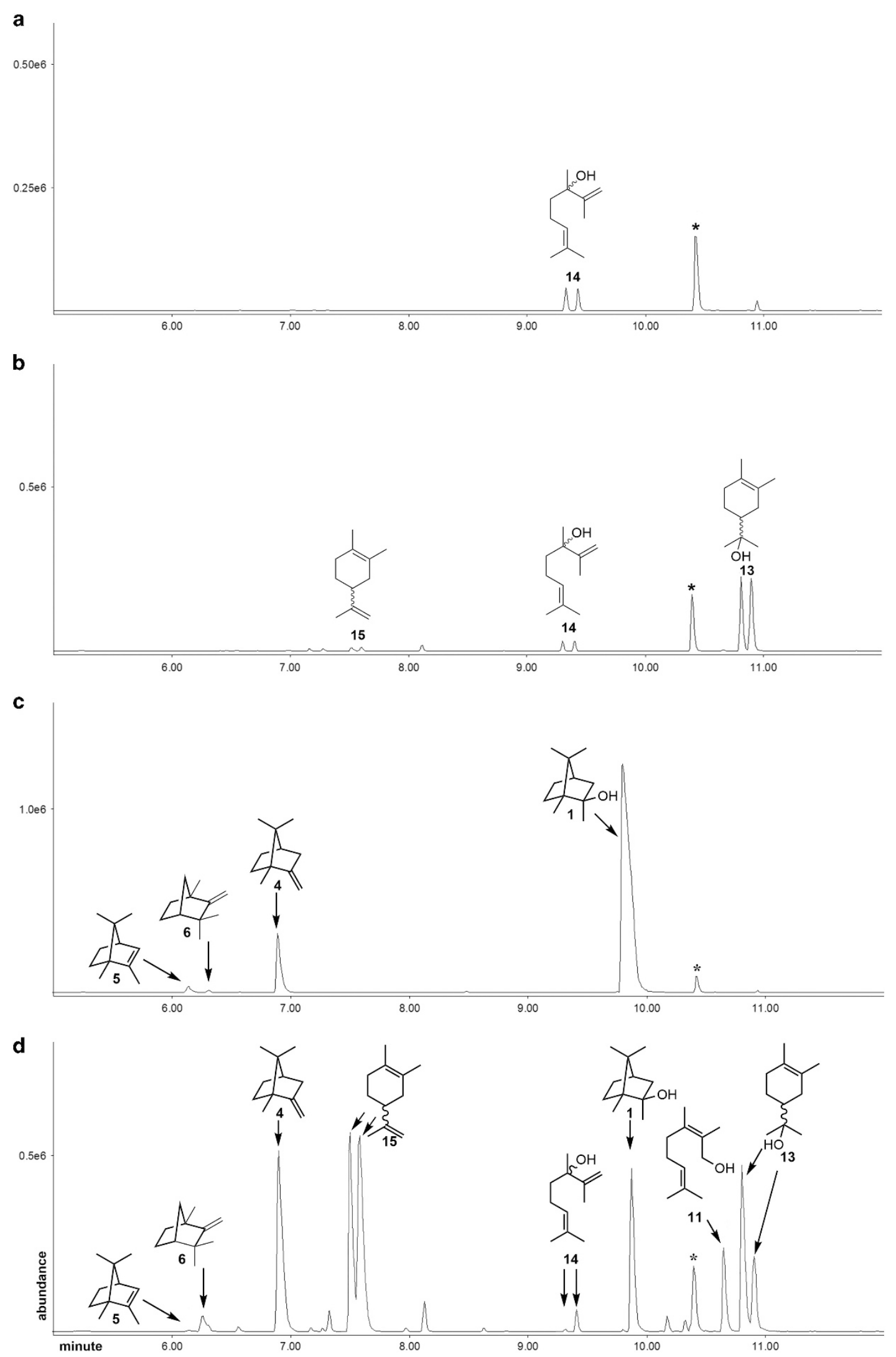

Figure 5 Chiral GC-MS analysis (total ion current, TIC) of incubations with methylisoborneol synthase (MIBS). (a) Control incubation of 2-MeGPP (3) in Mg2 ${ }^{+}$-containing buffer without MIBS. (b) Control incubation of 2-MeNPP (10) in $\mathrm{Mg}^{2+}$-containing buffer without MIBS. (c) Incubation of MIBS with 2-MeGPP (3). (d) Incubation of MIBS with 2-MeNPP (10). Previously unobserved product peaks are shown in red. Peaks denoted with *are geraniol internal standard. A full color version of this figure is available at the The Journal of Antibiotics journal online. 
Table 1 Distribution of products from the incubation of 2-MeGPP (3) and 2-MeNPP (10) with 2-MIB synthase

\begin{tabular}{|c|c|c|c|c|c|c|c|c|}
\hline \multirow[b]{2}{*}{ Substrate } & \multicolumn{8}{|c|}{ Product (\%) } \\
\hline & $M I B(1)$ & 4 & 5 & 6 & 13 & $( \pm)-15$ & 11 & 14 \\
\hline 2-MeGPP (3) & 89 & 10 & $<1$ & $<1$ & ND & ND & ND & ND \\
\hline 2-MeNPP (10) & 17 & 26 & $<1$ & $<1$ & $\begin{array}{c}10^{\mathrm{a}} \\
(10 / 0)^{\mathrm{b}}\end{array}$ & $\begin{array}{c}39^{a} \\
(17 / 22)^{b}\end{array}$ & 7 & $\begin{array}{c}<1^{\mathrm{a}} \\
(0 /<1)^{\mathrm{b}}\end{array}$ \\
\hline
\end{tabular}

Abrreviations: MeGPP, methylgeranyl disphosphate; MeNPP, methylneryl diphosphate;

MIB, methylisoborneol; ND, not detected.

apercent corrected for background $\mathrm{Mg}^{2+}$-catalyzed solvolysis.

bPercent first eluted peak/percent second eluted peak.

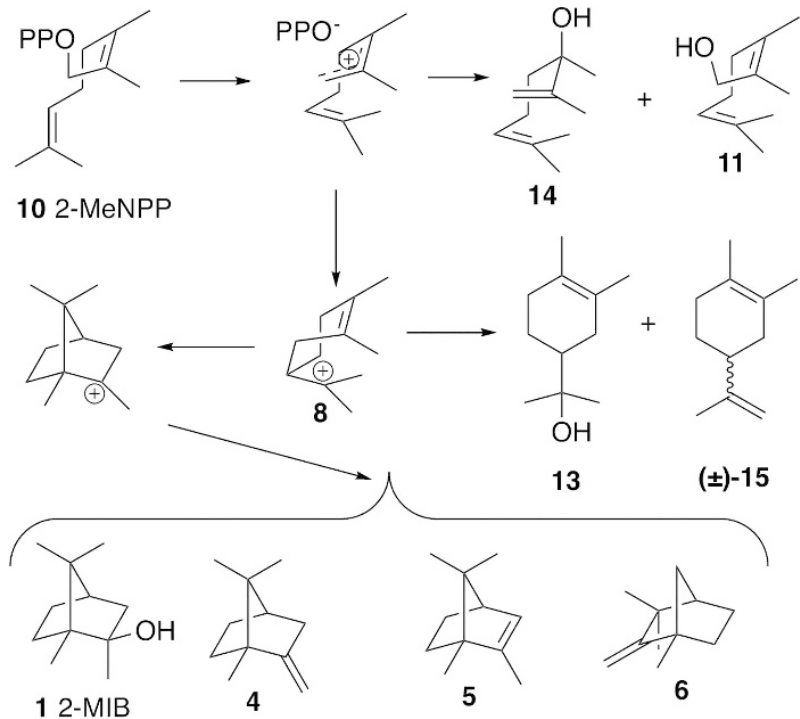

Figure 6 Mechanism of the methylisoborneol synthase (MIBS)-catalyzed transformation of 2-methylneryl diphosphate (2-MeNPP; 10).

constituting $>56 \%$ of the overall mixture were formed: monocyclic 2 -methyllimonene $(15,39 \%)$ and 2-methyl- $\alpha$-terpineol $(13,10 \%)$ along with the acyclic alcohols 2-methylnerol $(11,7 \%)$ and 2-methyllinalool (14, <1\%; Table 1, Figures $5 \mathrm{~d}$ and 6). Interestingly, correction for background $\mathrm{Mg}^{2+}$-catalyzed solvolysis of the 2-MeNPP substrate revealed that both 2-methyl- $\alpha$-terpineol (13) and 2-methyllinalool (14) were formed as single enantiomers, while 2 -methyllimonene (15) was generated as a racemic mixture. The absolute configurations of the enzymatically generated products $\mathbf{1 3}$ and $\mathbf{1 4}$ were not determined.

\section{Steady-state kinetics}

The steady-state kinetic parameters for the MIBS-catalyzed conversion of $\left[1-{ }^{3} \mathrm{H}\right]-2-\mathrm{MeNPP}(\mathbf{1 0})$ to total pentane-soluble homo-monoterpene products were determined and directly compared with those re-determined for the natural substrate $\left[1-{ }^{3} \mathrm{H}\right]-2-\mathrm{MeGPP}$ (3) under identical conditions (Table 2). While the turnover number, $k_{\text {cat }}$, for 2-MeNPP $\left(0.0045 \mathrm{~s}^{-1}\right)$ was $\sim 20$-fold lower than that for 2-MeGPP, the observed $k_{\text {cat }} / K_{\mathrm{m}}$, of $255 \mathrm{M}^{-1} \mathrm{~s}^{-1}$ for 2-MeNPP was only 4 -fold lower than that for 2-MeGPP $\left(1105 \mathrm{M}^{-1} \mathrm{~s}^{-1}\right)$, reflecting a partially compensating $\sim 5$-fold decrease in the observed $K_{\mathrm{m}}$ for 2 -MeNPP compared to 2 -MeGPP. ${ }^{8}$
Table 2 Steady-state kinetic parameters for total product formation from incubation with methylisoborneol synthase

\begin{tabular}{lccc}
\hline Substrate & $\mathrm{k}_{\text {cat }}\left(s^{-1}\right)$ & $\mathrm{K}_{m}(\mu \mathrm{m})$ & $\mathrm{k}_{\text {cat }} \mathrm{K}_{m}\left(M^{-1} s^{-1}\right)$ \\
\hline 2-MeGPP (3) & $0.105 \pm 0.007$ & $95 \pm 49$ & 1110 \\
2-MeNPP (10) & $0.0046 \pm 0.0003$ & $18 \pm 6$ & 255 \\
\hline
\end{tabular}

\section{DISCUSSION}

Although (Z)-2-MeNPP (10, 2-MeNPP) shows only a modest fourfold decrease in the specificity constant $k_{\mathrm{cat}} / K_{\mathrm{m}}$ compared to the natural substrate, the trans geometric isomer (E)-2-MeGPP (3), the substantially altered product distributions resulting from incubation of 2-MeNPP with 2-MIB synthase establish that 2-MeNPP is a poor surrogate for 2-MeGPP. Thus, while 2-MeNPP could, in principle, be ionized to the same cisoid allylic cation-pyrophosphate ion pair as the presumptive intermediate (3R)-2-methyllinalyl diphosphate (7, 2-MeLPP), in practice incubation of 2-MeNPP with 2-MIB synthase generated a substantially altered array of bicyclic and monocylic homo-monoterpene products. Thus, although cyclization of 2-MeNPP generated only a single enantiomer of each of the four natural bicyclic natural products, 2-MIB (1), 2-methylenebornane (4), 2-methylbornene (5) and 1 -methylcamphene (6), the relative proportion of $\mathbf{1}$ was reduced more than fivefold from $89 \%$ to $17 \%$ of the total product mixture, only partially offset by an increase in the fraction of 2-methylenebornane (4) which increased from 10 to $26 \%$ (Table 1). The majority of the product mixture resulting from the MIBS-catalyzed cyclization of 2-MeNPP (10) consisted of the anomalous monocyclic homo-monoterpenes ( \pm )-2methyllimonene $(15,39 \%)$ and a single enantiomer of 2 -methyl- $\alpha$ terpineol $(\mathbf{1 3}, 10 \%)$, as well as the acylic derivatives 2-methylnerol (11, $7 \%)$ and a single enantiomer of 2-methyllinalool $(14,<1 \%)$. Interestingly, while 13, 14 and 15 are not detected among the products of the in vitro incubation of 2-MIB synthase with 2-MeGPP, all three of these homo-monoterpenes, of unspecified chiral purity, have been reported as minor components of the volatile head-space extracts of a small number of 2-MIB-producing actinomycetes. ${ }^{19,20}$

Substantial changes in product distribution have been reported when the cis isomer neryl diphosphate is incubated with monoterpene synthases in place of the natural trans allylic substrate, geranyl diphosphate. For example, in spite of only minor differences in overall $k_{\text {cat }}$, incubation $(+)-\mathrm{BPP} /(+)$-pinene synthase with NPP instead of GPP resulted in a decrease in the proportion of the bicyclic monoterpenes $\alpha$-pinene and camphene from $79 \%$ to $23 \%$, while the fraction of the monocyclic products limonene and $\alpha$-terpinolene exhibited a substantial increase from 15 to $71 \%$ of total monoterpenes. ${ }^{21}$ The enhanced formation of abortive cyclization products is almost certainly the consequence of aberrant positioning of 2-MeNPP and NPP in the active sites of the respective homomonoterpene or monoterpene synthases, compared to the binding of the native substrates 2-MeGPP or GPP.

The fact that cyclization of 2-MeNPP by 2-MIB synthase generates the naturally occurring enantiomers of 2-MIB (1), 2-methylenebornane (4), 2-methylbornene (5) and 1-methylcamphene (6) indicates that at least a portion of this anomalous substrate is bound to MIBS in a position and conformation that is compatible with, but certainly not identical to, the precise folding of the natural substrate 2-MeGPP (3) and the derived intermediate (3R)-2-MeLPP (7) in the MIBS active site. Although the 2-methyllimonene (15) and 2-methyl- $\alpha$-terpineol (13) formed from 2 -MeNPP are both formally derivable from a common 2-methyl- $\alpha$-terpinyl cation, the fact that $\mathbf{1 5}$ is generated as a racemic mixture while $\mathbf{1 3}$ is produced as 
a single enantiomer indicates clearly that they cannot both be derived from a single-monocyclic carbocation intermediate. The surrogate 2-MeNPP substrate therefore must be bound in at least two distinct conformations during MIBS-catalyzed cyclization. The fact that only a single enantiomer of each of the bicyclic, monocyclic and acyclic alcohols 1, 13 and 14 is produced by incubation of 2-MeNPP with MIBS also suggests that the same bound water molecule is responsible for quenching the corresponding carbocation intermediates.

\section{EXPERIMENTAL PROCEDURE}

\section{Materials}

Reagents and solvents were purchased from Sigma-Aldrich (St Louis, MO, USA) or Fisher Scientific (Waltham, MA, USA), were of the highest quality available, and were used without further purification. $\mathrm{NaBH}_{3} \mathrm{~T}$ solution $\left(6 \mu \mathrm{mol}, 80 \mathrm{Ci} \mathrm{mmol}^{-1}\right.$ specific activity, $500 \mathrm{mCi}$ total in $1 \mathrm{ml}$ of $0.01 \mathrm{~N}$ $\mathrm{NaOH}$ ) was purchased from American Radiolabeled Chemicals (St Louis, MO, USA). Isopropylthio-D-galactopyranoside was purchased from Invitrogen (Waltham, MA, USA). Ni-NTA affinity resin was from Qiagen (Valencia, CA, USA). Amicon Ultra Centrifugal Filter Units (Amicon Ultra-15 10000 MWCO) were purchased from Millipore (St Charles, MO, USA). Purified SCO7700 protein was overexpressed and purified as previously described. ${ }^{7}$

\section{Methods}

GC - MS analyses were performed using an Agilent 5977A Series GC/MSD instrument (70 eV, electron impact), a $1-\mu \mathrm{l}$ injection volume, and a $3 \mathrm{~min}$ solvent delay. Achiral GC - MS conditions used an HP-5 ms capillary column $(0.25 \mathrm{~mm}$ ID $\times 30 \mathrm{~m}$ length $\times 0.25 \mu \mathrm{m}$ film, Agilent Technologies (Santa Clara, CA, USA)) and a temperature program with a 2 min hold at $60{ }^{\circ} \mathrm{C}$, a $20^{\circ} \mathrm{C} \mathrm{min}{ }^{-1}$ increment to $280{ }^{\circ} \mathrm{C}$, followed by a 2 min hold at $280{ }^{\circ} \mathrm{C}$. Chiral GC - MS separations were preformed using a CP-ChiralSil-Dex column $(0.32 \mathrm{~mm}$ ID $\times 25 \mathrm{~m}$ length $\times 0.25 \mu \mathrm{m}$ film, Agilent $)$ and a temperature program with a $1 \mathrm{~min}$ hold at $50^{\circ} \mathrm{C}$, a $10^{\circ} \mathrm{C} \mathrm{min}-1$ increment to $200{ }^{\circ} \mathrm{C}$, followed by a $1 \mathrm{~min}$ hold at $200^{\circ} \mathrm{C}$. Compounds detected by GC - MS were directly compared to their authentic standards using the MassFinder 4.2.1 program (http://www.massfinder.com). Retention indices were measured using $\mathrm{C}_{8}-\mathrm{C}_{20}$ and $\mathrm{C}_{10}-\mathrm{C}_{40}$ alkane standards.

LC-MS analyses were performed using a Finnigan LXQ LC-MS instrument in negative (electrospray ionization ESI) mode, with a Waters Symmetry $\mathrm{C}_{18}$ column ( $35 \mu \mathrm{M}, 2.1 \times 50 \mathrm{~mm})$, a $10 \mu \mathrm{l}$ injection volume and $1 \mathrm{~min}$ signal delay. The method used was a 1 min isocratic gradient of 95:5 water:acetonitrile, a gradient to $5: 95$ water:acetonitrile over $5 \mathrm{~min}$, followed by isocratic elution with 5:95 water:acetonitrile for 9 min. NMR spectra were obtained using a Bruker Avance AV400 NMR spectrometer operating at a $400 \mathrm{MHz}{ }^{1} \mathrm{H}$ frequency or a Bruker Avance AV300 NMR spectrometer operating at a $300 \mathrm{MHz}{ }^{1} \mathrm{H}$ frequency. Liquid scintillation counting was performed using a BeckmanCoulter LS6500 scintillation counter and Opti-Flour scintillation cocktail from Perkin-Elmer (Waltham, MA, USA).

All proteins were handled at $4{ }^{\circ} \mathrm{C}$ unless otherwise stated. Protein concentrations were determined according to the method of Bradford with bovine serum albumin as the standard. ${ }^{22}$ Protein purity was estimated using SDS PAGE gel electrophoresis and visualized using Coomassie Blue stain according to the method of Laemmli. ${ }^{23}$

\section{2-methylneryl diphosphate (10)}

The cis isomer 2-methylnerol (11) was obtained by preparation of a 1:1 mixture of 2-methylnerol (11) to 2-methylgeraniol (12) and separation of the isomeric alcohols using column chromatography as previously described. ${ }^{7}$ The purified 2methylnerol was then converted to 2-MeNPP (10) using the method developed by Poulter. ${ }^{24}$ Triphenylphosphine $(1.27 \mathrm{~g}, 4.75 \mathrm{mmol})$ was added to a stirring solution of 2-methylnerol $(11 ; 200 \mathrm{mg}, 1.19 \mathrm{mmol})$ in anhydrous $\mathrm{CCl}_{4}(16 \mathrm{ml})$ and the mixture was refluxed for $24 \mathrm{~h}$. The solvent was evaporated in vacuo and the resulting slurry was triturated with pentane $(30 \mathrm{ml})$ and filtered by suction filtration. Concentration of the pentane filtrate at room temperature provided crude 2-methylneryl chloride, which was used in the subsequent reaction without further purification. Tris(tetrabutylammonium) hydrogen pyrophosphate $(1.58 \mathrm{~g}$,
$2.38 \mathrm{mmol}$ ) was added to the 2-methylneryl chloride in dry $\mathrm{CH}_{3} \mathrm{CN}(10 \mathrm{ml})$ under nitrogen and the reaction was stirred at room temperature for 2 days. The solvent was removed under a gentle stream of $\mathrm{N}_{2}$ to give a viscous, light brown liquid, followed by the addition of $30 \mathrm{ml}$ of $0.05 \mathrm{M} \mathrm{KHCO}_{3}$ and extraction with ether $(5 \times 5 \mathrm{ml})$ to remove organic impurities. The resulting aqueous phase was applied to a DEAE-Sephadex column that had been equilibrated with $0.05 \mathrm{M}$ $\mathrm{KHCO}_{3}$. After loading, the column was washed with $100 \mathrm{ml}$ of $0.05 \mathrm{M} \mathrm{KHCO}_{3}$ and eluted with a linear gradient of $0.05 \mathrm{M} \mathrm{KHCO}_{3}(300 \mathrm{ml})$ to $1.0 \mathrm{M} \mathrm{KHCO}_{3}$ $(300 \mathrm{ml})$ at a flow rate of $2.0 \mathrm{ml} \mathrm{min}{ }^{-1}$. Fractions $(35 \mathrm{ml})$ were collected and lyophilized, and those containing 2-MeNPP (10) were dissolved in a minimum amount of $\mathrm{dH}_{2} \mathrm{O}$ and applied to a CHP-20P column equilibrated with $\mathrm{dH}_{2} \mathrm{O}$. The column was run with a stepwise gradient of $100 \mathrm{ml}$ of $\mathrm{dH}_{2} \mathrm{O}, 100 \mathrm{ml}$ of $5 \%$ aqueous $\mathrm{CH}_{3} \mathrm{CN}, 100 \mathrm{ml}$ of $10 \%$ aqueous $\mathrm{CH}_{3} \mathrm{CN}, 100 \mathrm{ml}$ of $20 \%$ aqueous $\mathrm{CH}_{3} \mathrm{CN}$ in $\mathrm{H}_{2} \mathrm{O}$ and $100 \mathrm{ml}$ of $60 \%$ aqueous $\mathrm{CH}_{3} \mathrm{CN}$ in $\mathrm{H}_{2} \mathrm{O}$. Fractions were lyophilized to yield $105 \mathrm{mg}$ of 2-MeNPP (10) as a pale yellow solid (27\% yield). $\mathrm{R}_{f}=0.35$ (6:3:1 propanol to $\mathrm{NH}_{4} \mathrm{OH}$ to water). ${ }^{1} \mathrm{H}$ NMR $\left(400 \mathrm{MHz}, \mathrm{D}_{2} \mathrm{O}\right) \delta 5.08$ (t, $J=4.5 \mathrm{~Hz}, 1 \mathrm{H}, \mathrm{H}-6), 4.31$ (d, J=4.0 Hz, 2H, H-1), 2.05 (m, 2H, H-4), 1.98 (m, 2H, H-5), 1.61 (s, 3H, H-11), 1.58 (s, 3H, H-9), 1.55 (s, 3H, H-10), 1.49 (s, 3H, H-8). ${ }^{31} \mathrm{P}\left\{{ }^{1} \mathrm{H}\right\}$ NMR $\left(161 \mathrm{MHz}, \mathrm{D}_{2} \mathrm{O}\right) \delta-6.6\left(\mathrm{~d}, J=22.5 \mathrm{~Hz}, \mathrm{P}_{\beta}\right)$, $-10.4\left(\mathrm{~d}, J=22.5 \mathrm{~Hz}, \mathrm{P}_{\alpha}\right) \cdot{ }^{13} \mathrm{C}\left\{{ }^{1} \mathrm{H}\right\} \operatorname{NMR}\left(75 \mathrm{MHz}, \mathrm{D}_{2} \mathrm{O}\right): \delta 16.1\left(\mathrm{CH}_{3}\right), 17.0$ $\left(\mathrm{CH}_{3}\right), 18.2\left(\mathrm{CH}_{3}\right), 24.9\left(\mathrm{CH}_{3}\right), 26.6\left(\mathrm{CH}_{2}\right), 33.3\left(\mathrm{CH}_{2}\right), 66.3\left(\mathrm{CH}_{2}\right), 124.1(\mathrm{CH})$, 133.8 (C), 135.6 (C), 160.3 (C). LC-MS (neg ion ESIMS) $\mathrm{m} / \mathrm{z} 326.99$.

$\left[1-{ }^{3} \mathrm{H}\right]-2-$ Methylneryl diphosphate $\left(\left[1-{ }^{3} \mathrm{H}\right]-10\right)$ and $\left[1-{ }^{3} \mathrm{H}\right]-2-$ methylgeranyl diphosphate $\left(\left[1-{ }^{3} \mathrm{H}\right]-3\right)$

A $1.0 \mathrm{ml}$ solution of $\mathrm{NaBH}_{3} \mathrm{~T}\left(6 \mu \mathrm{mol}, 80 \mathrm{Ci} \mathrm{mmol}^{-1}, 500 \mathrm{mCi}\right.$ in $1 \mathrm{ml}$ of $0.01 \mathrm{~N} \mathrm{NaOH}$ ) was added to a round bottom flask. The reagent vial was rinsed with $4 \times 1 \mathrm{ml}$ of $0.01 \mathrm{~N} \mathrm{NaOH}$ which was added to the solution and then placed under argon, cooled in an ice bath. An ice-cold 1:1 mixture of 2-methylneral to 2-methylgeranial $(425 \mathrm{mg}, 2.55 \mathrm{mmol}$ ), freshly prepared as previously described, ${ }^{7}$ in dry EtOH $(7.5 \mathrm{ml})$ was added slowly over $10 \mathrm{~min}$. The reaction was stirred at $0{ }^{\circ} \mathrm{C}$ for $3 \mathrm{~h}$, and the remaining aldehydes were reduced by the slow addition of unlabeled $\mathrm{NaBH}_{4}$ powder $(75 \mathrm{mg}, 2.0 \mathrm{mmol})$. The resulting solution was warmed to room temperature and stirred overnight. After a total of $22 \mathrm{~h}$, the reaction was quenched by careful transfer of the mixture into ice-cold half-saturated aqueous $\mathrm{NH}_{4} \mathrm{Cl}(10 \mathrm{ml})$. The aqueous layer was extracted with $\mathrm{Et}_{2} \mathrm{O}(5 \times 10 \mathrm{ml})$ and the combined extracts were dried, filtered and concentrated to give $310 \mathrm{mg}$ of yellowish liquid (72\% crude chemical yield, $375 \mathrm{mCi}, 75 \%$ radiochemical yield). The residue was purified by flash chromatography on $10 \%(\mathrm{w} / \mathrm{w}) \mathrm{AgNO}_{3}$-impregnated silica gel (EtOAc/hexanes (1:9); $4 \times 16-\mathrm{cm}$ column) giving $100 \mathrm{mg}$ of $\left[1-{ }^{3} \mathrm{H}\right]-2$-methylnerol $\left(\left[1-{ }^{3} \mathrm{H}\right]-11,25 \%\right.$ chemical yield, $128 \mathrm{mCi}, 26 \%$ radiochemical yield $)$ and $210 \mathrm{mg}$ of $\left[1-{ }^{3} \mathrm{H}\right]-2$-methylgeraniol $\left(\left[1-{ }^{3} \mathrm{H}\right]-12,48 \%\right.$ chemical yield, $247 \mathrm{mCi}$, $49 \%$ radiochemical yield). The purified $\left[1-{ }^{3} \mathrm{H}\right]-2$-methylnerol $\left(\left[1-{ }^{3} \mathrm{H}\right]-11\right)$ and $\left[1-{ }^{3} \mathrm{H}\right]$ 2-methylgeraniol $\left(\left[1-{ }^{3} \mathrm{H}\right]-12\right)$ were individually converted to the corresponding diphosphate esters using the same method employed for the synthesis of unlabeled 10 and 3 to yield $98 \mathrm{mg}$ of $\left[1-{ }^{3} \mathrm{H}\right]-2-\mathrm{MeNPP}$ $\left(\left[1-{ }^{3} \mathrm{H}\right]-10\right), 37 \%$ chemical yield, $6.2 \mathrm{mCi}$ total radioactivity, $2.2 \%$ radiochemical yield, $28 \mathrm{mCi} / \mathrm{mmol}$ specific activity) and $200.1 \mathrm{mg}$ of $\left[1-{ }^{3} \mathrm{H}\right] 2$ MeGPP $\left(\left[1-{ }^{3} \mathrm{H}\right]-3, \quad 36 \%\right.$ chemical yield, $105 \mathrm{mCi}$ total radioactivity, $36 \%$ radiochemical yield and $232.8 \mathrm{mCi} / \mathrm{mmol}$ specific activity). The NMR spectra of $\left(\left[1-{ }^{3} \mathrm{H}\right]-10\right.$ and of $\left[1-{ }^{3} \mathrm{H}\right]-3$ matched those of unlabeled 10 and $3 .^{7}$

\section{2-Methyl- $\alpha$-terpineol (13)}

p-TsOH (40 mg, $0.20 \mathrm{mmol}$ ) was added to a solution of 2-methylnerol (200 mg, $1.19 \mathrm{mmol}$ ) in $10 \mathrm{ml}$ of $\mathrm{MeNO}_{2}$. The reaction mixture was stirred at $\mathrm{rt}$ for $1 \mathrm{~h}$ and monitored by TLC (5:1 hexanes/ethyl acetate). The crude product was concentrated in vacuo and purified by column chromatography (5:1 hexanes/ethyl acetate) yielding $44 \mathrm{mg}$ of 2 -methyl- $\alpha$-terpineol $(13,22 \%$ yield). The NMR data matched those previously reported for 13 prepared by an alternative method. ${ }^{19}{ }^{1} \mathrm{H}$ NMR $\left(400 \mathrm{MHz}, \mathrm{CDCl}_{3}\right) \delta 1.98$, (m, 2H, H-6), 1.85 (m, 2H, H-3), 1.72 (m, 1H, H-5), 1.65 (s, 6H, H-10, 11), 1.48 (m, 2H, H-4), $1.22(\mathrm{~s}, 6 \mathrm{H}, \mathrm{H}-8,9) .{ }^{13} \mathrm{C}\left\{{ }^{1} \mathrm{H}\right\}$ NMR $\left(100 \mathrm{MHz}, \mathrm{CDCl}_{3}\right) \delta 19.4\left(\mathrm{C}-9, \mathrm{CH}_{3}\right)$, $19.5\left(\mathrm{C}-8, \mathrm{CH}_{3}\right), 24.3\left(\mathrm{C}-3, \mathrm{CH}_{2}\right), 26.3\left(\mathrm{C}-6, \mathrm{CH}_{2}\right), 27.3\left(\mathrm{C}-4, \mathrm{CH}_{2}\right)$, $32.7\left(\mathrm{C}-11, \mathrm{CH}_{3}\right), 33.2\left(\mathrm{C}-10, \mathrm{CH}_{3}\right), 45.8(\mathrm{C}-5, \mathrm{CH}), 72.9(\mathrm{C}-7, \mathrm{C})$, 125.0 (C-2, C), 125.8 (C-1, C). GC-MS (EI) $\mathrm{m} / z$ 168.1. 
Incubations with 2-methylisoborneol synthase (SCO7700)

Purified 2-MIB synthase $(\mathrm{SCO} 7700 ; 10 \mu \mathrm{M})$ was added to a glass test tube containing $3.0 \mathrm{ml}$ of assay buffer (50 mM PIPES, $100 \mathrm{~mm} \mathrm{NaCl}, 15 \mathrm{~mm} \mathrm{MgCl}_{2}$, $5 \mathrm{~mm} \beta$-mercaptoethanol and 20\% glycerol, $\mathrm{pH} 6.7$ ) and $60 \mu \mathrm{m}$ of either 2-MeNPP (10) or 2-MeGPP (3). The enzymatic reaction mixture was overlaid with $3.0 \mathrm{ml}$ of pentane and incubated at $30^{\circ} \mathrm{C}$ for $2 \mathrm{~h}$. The enzymatic products were extracted with $3 \times 3.0 \mathrm{ml}$ of pentane and the organic extracts dried over $\mathrm{Na}_{2} \mathrm{SO}_{4}$, filtered, concentrated in vacuo to $100 \mu \mathrm{l}$ and analyzed by GC-MS. Control reactions, measuring the background $\mathrm{Mg}^{2+}$-catalyzed hydrolysis of each substrate, were conducted similarly as above minus the addition of SCO7700 to the incubation mixture. An internal geraniol standard (25 pmol) was added to the organic extracts of the enzymatic and control incubations after extraction.

\section{Steady-state kinetics}

Kinetic analyses were performed in $1 \mathrm{ml}$ of assay buffer (50 mм PIPES, $15 \mathrm{~mm}$ $\mathrm{MgCl}_{2}, 100 \mathrm{~mm} \mathrm{NaCl}, 5 \mathrm{~mm} \beta$-mercaptoethanol, $20 \%$ glycerol and $\mathrm{pH} 6.7$ ) with either 2-MeNPP $(\mathbf{1 0}, 10-500 \mu \mathrm{M})$ and $\left[1-{ }^{3} \mathrm{H}\right]-2-\mathrm{MeNPP}\left(\left[1-{ }^{3} \mathrm{H}\right]-\mathbf{1 0}\right.$, $8.4 \mathrm{Ci} / \mathrm{mol})$ or 2 -MeGPP $(3,10-500 \mathrm{~mm})$ and $\left[1-{ }^{3} \mathrm{H}\right] 2-\mathrm{MeGPP}\left(\left(\left[1-{ }^{3} \mathrm{H}\right]-3\right.\right.$, $\left.16.5 \mathrm{Ci} \mathrm{mol}^{-1}\right)$. The reactions were initiated by the addition of $0.20 \mu \mathrm{M}$ of SCO7700 protein, overlaid with $1 \mathrm{ml}$ of pentane and incubated at $30^{\circ} \mathrm{C}$ for $17 \mathrm{~min}$. The reactions were quenched by the addition of $75 \mu \mathrm{l}$ of $500 \mathrm{~mm}$ EDTA $(\mathrm{pH} 8.0)$ and vortexing for $30 \mathrm{~s}$. The pentane layer was loaded onto a silica column $(2 \mathrm{~cm})$ in a Pasteur pipette and forced through with a stream of nitrogen into a scintillation vial containing $7 \mathrm{ml}$ of Opti-Fluor. The enzymatic reaction mixture was extracted 3 more times with $1 \mathrm{ml}$ of ether, and all organic extracts were passed through the silica column and collected. The combined extracts were assayed by liquid scintillation counting using a Beckman-Coulter LS6500 scintillation counter. Kinetic constants were calculated using the program Kaleidagraph 4.0 and were fit to the Michaelis-Menten equation. Reported standard deviations in the steady-state kinetic parameters represent the calculated statistical errors in the nonlinear, least squares regression analysis.

\section{CONFLICT OF INTEREST}

The authors declare no conflict of interest.

\section{ACKNOWLEDGEMENTS}

This work was supported by NIH grant GM30301 (DEC). We thank Tun-Li Shen for assistance with MS and Russell Hopson for assistance with NMR spectroscopy.

1 Gerber, N. N. A volatile metabolite of actinomycetes, 2-methylisoborneol. J. Antibiot 22, 508-509 (1969).

2 Jüttner, F. \& Watson, S. B. Biochemical and ecological control of geosmin and 2 methylisoborneol in source waters. Appl. Environ. Microbiol. 73, 4395-4406 (2007).
3 Giglio, S., Chou, W. K., Ikeda, H., Cane, D. E. \& Monis, P. T. Biosynthesis of 2-methylisoborneol in cyanobacteria. Environ. Sci. Technol. 45, 992-998 (2011).

4 Dickschat, J. S. et al. Biosynthesis of the off-flavor 2-methylisoborneol by the myxobacterium Nannocystis exedens. Angew. Chem. Int. Ed. Engl. 46, 8287-8290 (2007).

5 Yamada, Y. et al. Terpene synthases are widely distributed in bacteria. Proc. Natl Acad. Sci. USA 112, 857-862 (2015).

6 Komatsu, M., Tsuda, M., Omura, S., Oikawa, H. \& Ikeda, H. Identification and functional analysis of genes controlling biosynthesis of 2-methylisoborneol. Proc. Nat Acad. Sci. USA 105, 7422-7427 (2008).

7 Wang, C. M. \& Cane, D. E. Biochemistry and molecular genetics of the biosynthesis of the earthy odorant methylisoborneol in Streptomyces coelicolor. J. Am. Chem. Soc. 130, 8908-8909 (2008).

8 Wang C. M. \& Cane, D. E. Biochemistry and molecular genetics of the biosynthesis of the earthy odorant methylisoborneol in Streptomyces coelicolor (corrn to J. Am. Chem. Soc. 130, 8908 (2008)). J. Am. Chem. Soc. 132, 9509 (2010).

9 Chou, W. K., Ikeda, H. \& Cane, D. E. Cloning and characterization of Pfl 1841, a 2-methylenebornane synthase in Pseudomonas fluorescens PfO-1. Tetrahedron 67, 6627-6632 (2011).

10 Wise, M. L. \& Croteau, R. in Comprehensive Natural Products Chemistry, Vol. 2 (ed. D. E. Cane) 97-153 (Elsevier, New York, NY, USA, 1999).

11 Croteau, R., Satterwhite, D. M., Cane, D. E. \& Chang, C. C. Biosynthesis of monoterpenes: enantioselectivity in the enzymatic cyclization of $(+)$ - and $(-)$-linalyl pyrophosphate to (+)- and (-)-bornyl pyrophosphate. J. Biol. Chem. 261, 13438-13445 (1986).

12 Croteau, R., Satterwhite, D. M., Cane, D. E. \& Chang, C. C. Enantioselectivity in the enzymatic cyclization of (+)- and ( - )-linalyl pyrophosphate to $(+)$ - and ( - )-pinene and (+)- and ( - )-camphene. J. Biol. Chem. 263, 10063-10071 (1988).

13 Wise, M. L., Savage, T. J., Katahira, E. \& Croteau, R. Monoterpene synthases from common sage (Salvia officinalis). cDNA isolation, characterization, and functional expression of (+)-sabinene synthase, 1,8-cineole synthase, and (+)-bornyl diphosphate synthase. J. Biol. Chem. 273, 14891-14899 (1998).

14 Koksal, M., Chou, W. K., Cane, D. E. \& Christianson, D. W. Structure of 2-methylisoborneol synthase from Streptomyces coelicolor and implications for the cyclization of a noncanonical C-methylated monoterpenoid substrate. Biochemistry 51, 3011-3020 (2012).

15 Koksal, M., Chou, W. K., Cane, D. E. \& Christianson, D. W. Unexpected reactivity of 2-fluorolinalyl diphosphate in the active site of crystalline 2-methylisoborneol synthase. Biochemistry 52, 5247-5255 (2013).

16 Whittington, D. A. et al. Bornyl diphosphate synthase: structure and strategy for carbocation manipulation by a terpenoid cyclase. Proc. Natl Acad. Sci. USA 99, 15375-15380,( (2002).

17 Hyatt, D. C. et al. Structure of limonene synthase, a simple model for terpenoid cyclase catalysis. Proc. Natl Acad. Sci. USA 104, 5360-5365 (2007).

18 George-Nascimento, C., Pont-Lezicka, R. \& Cori, O. Nonenzymic formation of nerolidol from farnesyl pyrophosphate in the presence of bivalent cations. Biochem. Biophys. Res. Commun. 45, 119-124 (1971).

19 Brock, N. L., Ravella, S. R., Schulz, S. \& Dickschat, J. S. A detailed view of 2-methylisoborneol biosynthesis. Angew. Chem. Int. Ed. Engl. 52, 2100-2104 (2013).

20 Citron, C. A., Barra, L., Wink, J. \& Dickschat, J. S. Volatiles from nineteen recently genome sequenced actinomycetes. Org. Biomol. Chem. 13, 2673-2683 (2015).

21 Croteau, R. \& Satterwhite, D. M. Biosynthesis of monoterpenes. Stereochemical implications of acyclic and monocyclic olefin formation by (+)- and (-)-pinene cyclases from sage. J. Biol. Chem. 264, 15309-15315 (1989).

22 Bradford, M. A rapid and sensitive method for the quantitation of microgram quantitites of protein utilizing the principle of protein-dye binding. Anal. Biochem. 72 , 248-254 (1976).

23 Laemmli, U. K. Cleavage of structural proteins during the assembly of the head of Bacteriophage T4. Nature (London) 227, 680-685 (1970).

24 Davisson, V. J. et al. Phosphorylation of isoprenoid alcohols. J. Org. Chem. 51, 4768-4779 (1986).

Supplementary Information accompanies the paper on The Journal of Antibiotics website (http://www.nature.com/ja). 